\title{
A Hierarchical Reduced Markov Model for Reliability Evaluation of Phased-Mission Systems
}

\author{
Hua Yan' ${ }^{1}$, Pu Long Cui*1, Yi Sheng Wang ${ }^{1}$, Bi Xing Lii ${ }^{2}$, Fei Wan ${ }^{1}$ and De Li \\ ${ }^{1}$ Department of Military Logistics, China \\ ${ }^{2}$ Department of Oil Management, China
}

Received: 制: November 23, 2018; Published: 制: November 30, 2018

*Corresponding author: Pu Long Cui, Department of Military Logistics, Chongqing, China

\begin{abstract}
This paper presents a reduced Markov model to evaluate the Phased-Mission System (PMS) reliability. The time cost and storage requirement are very huge using traditional Markov model to analyze the reliability of PMS as the number of components increases to a large scale. The hierarchical reduction approach proposed in this paper can account for the PMS which is composed of different level components. Such as thrust equipment of an aircraft can be further decomposed into compressor and turbine runner. The reduced Markov model by hierarchical reduction will have a smaller number of system states than the traditional model. Finally, the effectiveness and correctness of our approach is analyzed by comparing with the traditional Markov method.
\end{abstract}

\section{Nomenclature}

\section{Introduction}

Many practical systems, such as those encountered in electronic, aerospace and nuclear power have multiple phases of operations during their mission and are generally referred to as Phased-Mission System (PMS) [1]. Compared with general system, the reliability evaluation of PMS is much more complicated because of the components dynamic behavior as well as their dependence across the phases. Considerable efforts have been made in the analysis of PMS. Generally, existing methods can be roughly classified into two groups: Analytical methods and simulation methods [2]. The analytical methods are further classified into combinatorial approaches, state-based approaches, hierarchical approaches and modularization approaches. The combinatorial approaches, especially the binary decision diagram-based algorithm $[3,4]$, exploit the Boolean algebra to reduce the model scale, which are effective in evaluating non-repairable PMS. The state-based approaches are mainly based on Markov model [5,6], which suffers from the state-explosion problem when is applied to large-scale systems. The simulation methods typically offer greater generality in system representation, but they suffer from computational inefficiency and great computer memory consumption [7].

The above-mentioned methods mainly apply to small-scale PMSs due to the high computational complexity. This paper proposed a reduced Markov reliability model by using hierarchical reduction techniques. Compared with the traditional Markov model, the proposed method can efficiently reduce the number of system states, and avoid the well-known state-explosion problem. The remainder of the paper is organized as follows. Section 2 describes the hierarchical reduced Markov model. Section 3 illustrates our method with a specific example. Lastly, section 4 concludes the study and discusses future works.

\section{The Hierarchical Reduced Markov Model}

It is common that the real engineering systems provide hierarchical feature which can be used to advantage in the PMS reliability analysis. For example, thrust subsystem for an aircraft frequently effect all phases of a mission, maybe in different quantities, at each stage of flight [8]. Furthermore, the top-level subsystem can be decomposed into lower level components such as compressor and turbine runner. Such structure is common in many other engineering systems, not just those relating to aircraft. The hierarchical architecture is also a usual style in software reliability estimation, in which systems are decomposed into different layers according to the components' calling relations [9]. And it is also used to describe the interpreter relations of different layers in communication software. Accounting for hierarchical structure in the phase failure logic will enable a more reduced Markov model to be developed. One way of taking advantage of these features is using hierarchical reduction method which can reduce the state space of Markov model efficiently by subsystems replacement. The 
main idea of this method is generating a hypothetical component to replace the top-level subsystem. The components of subsystems are considered to be all in series or in parallel. Thus, the failure rate and repair rate of the hypothetical component can be derived by manipulation of the corresponding components' parameters in the subsystem. Also, it is important that the subsystems replaced need to be totally independent of all other sections of the mission failure fault tree.

The detailed process of the hierarchical reduction method is discussed taking a $n$ components subsystem for example. Let $\lambda_{i}$ and $\mu_{i}$ be the failure rates and repair rates of component $C_{i}$. Also, $\lambda_{s}$ and $\mu_{s}$ are the failure rate and repair rate of hypothetical component $C_{s}$ representing the subsystem.

1) if the $n$ components are series, $\lambda_{s}$ can be given as

$$
\lambda_{S}=\sum_{i=1}^{n} \lambda_{i}
$$

Let $A_{s}$ denote availability of the subsystem, where is defined by

$$
A_{S}=\frac{\mu_{S}}{\lambda_{S}+\mu_{S}}
$$

A series system is working when all of the components do not fail. If state 1 represents that all components are operational and $A_{1}$ is the steady-state availability of system in state 1 , then $A_{1}$ can be computed as follows.

$$
A_{1}=1 /\left(1+\sum_{i=1}^{n} \frac{\lambda_{i}}{\mu_{i}}\right)
$$

Since state 1 is the only working state in series system, it can be derived that. $A_{s}=A_{1}$ Moreover, according to Eq. (2) and (3), we have

$$
\mu_{S}=\sum_{i=1}^{n} \lambda_{i} / \sum_{i=1}^{n} \frac{\lambda_{i}}{\mu_{i}}
$$

From Eq. (1) and (4), we can use the hypothetical component $C_{s}$ with failure rate $\lambda_{s}$ and repair rate $\mu_{s}$ to replace the series subsystem.

2) if the $n$ components are parallel, $\mu_{s}$ can be given as

$$
\mu_{S}=\sum_{i=1}^{n} \mu_{i}
$$

For parallel system, the formula of availability $A_{s}$ is presented in [19] as follows.

$$
A_{S}=1-\prod_{i=1}^{n}\left(1-A_{i}\right)
$$

in which $A_{i}=\mu_{i} /\left(\lambda_{i}+\mu_{i}\right)$ is the availability of component $c_{i}$. Hence, using Eq. (2), (5) and (6), we obtain

$$
\lambda_{S}=\sum_{i=1}^{n} \mu_{i} \cdot\left(\prod_{i=1}^{n}\left(\frac{\lambda_{i}}{\lambda_{i}+\mu_{i}}\right)\right) /\left(1-\prod_{i=1}^{n}\left(\frac{\lambda_{i}}{\lambda_{i}+\mu_{i}}\right)\right)
$$

From Eq. (5) and (7), we can use the hypothetical component $c_{S}$ with failure rate $\lambda_{S}$ and repair rate $\mu_{s}$ to replace the parallel subsystem.

\section{Case Study}

In this section we describe a case study to verify the proposed reduced Markov model for PMS reliability analysis. The mission reliability of the PMS has been calculated by the traditional Markov method. The example PMS is a space flight Telemetry, Tracking and Control (TT\&C) system. The failure fault trees of the example TT\&C mission are shown in Figure 1, in which station1, station2, and station3 are composed of $\mathrm{C} 1 \sim \mathrm{C} 6, \mathrm{C} 7 \sim \mathrm{C} 8$, and $\mathrm{C} 9 \sim \mathrm{C} 10$ respectively. Assume that the failure rates and repair rates of components C1 C10 are unchanged across phases. The relevant parameters are detailed as follows: failure rates $\lambda_{i}=3.33 \times 10^{-3}$; repair rates $\mu_{i}=3.33 \times 10^{-2}$; phase transition time $t_{1}=20 \mathrm{~s}, t_{2}=120 \mathrm{~s}$ and $t_{3}=85 \mathrm{~s}$.

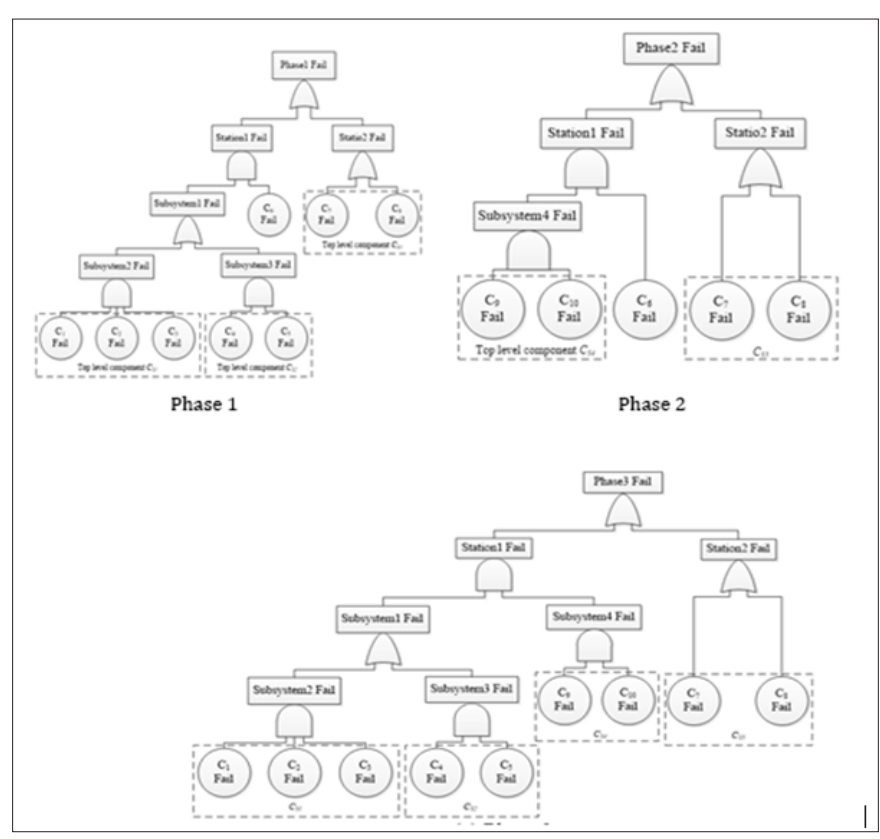

Figure 1: The failure fault tree of the three basic phases. 
By analyzing fault trees of the three basic phases, there are four subsystems can be reduce using hierarchical reduction method. These are $S_{1}$ with components $c_{1}, c_{2}$ and $c_{3}, S_{2}$ with components $c_{4}$ and $c_{5}, S_{3}$ with components $c_{7}$ and $c_{8}$, and $S_{4}$ with components $c_{9}$ and $c_{10}$. Therefore, the top level subsystems $S_{1}, S_{2}, S_{3}$ and $S_{4}$ can be replaced by four hypothetical components $c_{S 1}, c_{S 2}, c_{s 3}$ and $c_{S 4}$ respectively. For the series subsystem, failure rate and repair rate of the top-level components can be computed by using Eq. (1) and (4). Similarly, Eq. (5) and (7) can be used for the parallel subsystem. The computed parameters of the top-level hypothetical components are presented in Table 1. After hierarchical reduction, the new PMS phases configuration is shown in Figure 2.

Table 1: Computed parameters of the hypothetical components (unit: min).

\begin{tabular}{|c|c|c|c|}
\hline Parameters & $c_{S 1}$ & $c_{S 2}$ & $c_{S 3}$ \\
\hline Failure rates & $\lambda_{S 1}=7.519 \times 10^{-5}$ & $\lambda_{S 1}=5.556 \times 10^{-4}$ & $\lambda_{S 3}=6.667 \times 10^{-3}$ \\
\hline Repair rates $\mu_{i}$ & $\mu_{S 1}=0.1$ & $\mu_{S 2}=6.667 \times 10^{-2}$ & $\lambda_{S 1}=5.556 \times 10^{-4}$ \\
$s 3$ & $\mu_{S 2}=6.667 \times 10^{-2} 10 \quad 333 \times 10 \quad$ \\
\hline
\end{tabular}

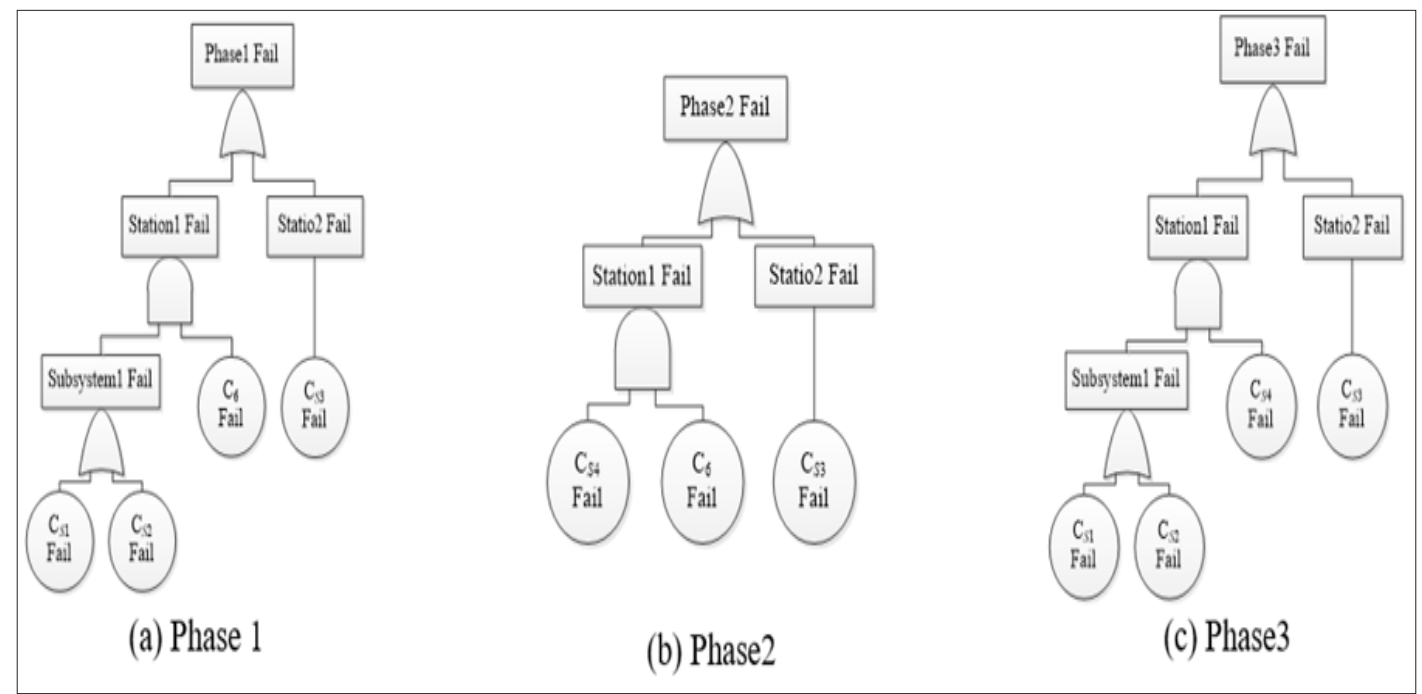

Figure 2: New phases configuration of the PMS after hierarchical reduction.

From Figure 2, we can derive that the number of reduced system states of the three phases are 16,8 , and 16 respectively. For the original PMS, these are 256,32, and 512 respectively. Because the number of components is decreased efficiently by using hypothetical components to replace the top-level subsystems, and it will slow down the increase in transition rate matrix scale. The PMS reliability and computation time of the traditional Markov method, and the hierarchical reduction method are presented in Table 2. The computing errors of each phase between traditional method and the hierarchical method are shown in Figure 3. Results in Table
2 indicate that there is an excellent match between the proposed method to the traditional Markov method, with relative error less than $1.19 \times 10-5$. Furthermore, the computation time of our method is less than the traditional Markov method. This is mainly because that the number of system states of simplified Markov model is less than that of traditional models. However, from Figure 3 we can see that the computing errors increase dramatically with the mission time. Thus, the hierarchical reduced Markov model is applicable for short duration PMS.

Table 2: Reliability and computation time of the example PMS.

\begin{tabular}{|c|c|c|c|}
\hline \multirow{2}{*}{} & \multicolumn{2}{|c|}{ Traditional Markov method } & \multicolumn{2}{c|}{ Proposed method } \\
\cline { 2 - 4 } & PMS reliability & Time cost(s) & PMS reliability \\
\hline Phase 1 & 0.997780243 & 0.015 & 0.99778001 \\
\hline Phase 2 & 0.984564486 & 0.063 & 0.98455638 \\
\hline Phase 3 & 0.975309577 & 0.320 & 0.97529770 \\
\hline
\end{tabular}




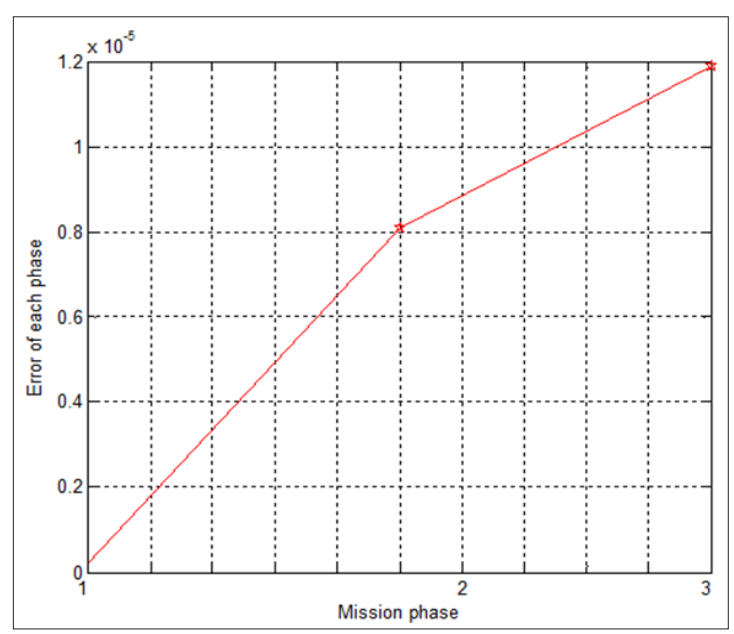

Figure 3: Computing errors between traditional method and the hierarchical method.

\section{Conclusion}

This paper presents a reduced Markov model using hierarchical reduction approach to evaluate the PMS reliability. The traditional Markov model suffers from the problem of huge transition rate matrix. Our approach takes advantage of PMS provide hierarchical feature, and an simplify the original Markov model of each phase by hierarchical reduction. In comparison to the traditional Markov approach, the proposed method achieves less time cost, also has good computation accuracy. However, the hierarchical process may cause computation errors. In future work, more attention will be addressed on error analysis, and efforts should be made to improve current method to achieve more accuracy and efficiency.

\section{ISSN: 2574-1241}

DOI: $10.26717 / B J S T R .2018 .11 .002128$

Pu Long Cui. Biomed J Sci \& Tech Res

This work is licensed under Creative Commons Attribution 4.0 License

Submission Link: https://biomedres.us/submit-manuscript.php

\section{Acknowledgement}

This research is funded by the National Nature Science Foundation of China with the Granted No. 71401172. The authors are grateful for the reviewer's helpful comments.

\section{References}

1. R Peng, QQ Zhai, LD Xing, J Yang (2016) Reliability analysis and optimal structure of series-parallel phased-mission systems subject to fault level coverage. IIE Transactions 48(8): 736-746.

2. JM Lu, XY Wu, Yiliu Liu, MaryAnn Lundteigen (2015) Reliability analysis of large phased-mission systems with repairable components based on success-state sampling. Reliability Engineering \& System Safety 142: 123-133.

3. YC Mo (2009) Variable ordering to improve BDDanalysis of phasedmission systems with multimode failures. IEEE Transactions on Reliability 58(1): 53-57.

4. LD Xing, G Levitin (2013) BDD-Based Reliability Evaluation of PhasedMission Systems with Internal/External Common-Cause Failures. Reliability Engineering \& System Safety 112(1): 145-153.

5. X Y Wu, H Yan, L R Li (2013) Numerical Method for Reliability Analysis of Phased-mission System using Markov Chains. Communications in Statistics - Theory and Methods 41(21): 3960-3973.

6. H Yan, K Wang, ZL Liu (2016) Reliability analysis of phased-mission system using Markov approach". Journal of Ordnance Equipment Engineering 37(6): 92-96.

7. $\mathrm{XY} \mathrm{Wu}, \mathrm{XY} W u(2015)$ Extended object-orient petri net model for mission reliability simulation of repairable pms with common causefailures. Reliability Engineering \& System Safety 136(C): 109-119.

8. JD Andrews, J Poole, W H Chen (2013) Fast mission reliability prediction for Unmanned Aerial Vehicles. Reliability Engineering \& System Safety 120: 3-9.

9. Y Wei, LB Wang, MQ Wang (2011) Software reliability analysis of Hierarchical architecture based on Markov model. Procedia Engineering 15: 2857-2861.

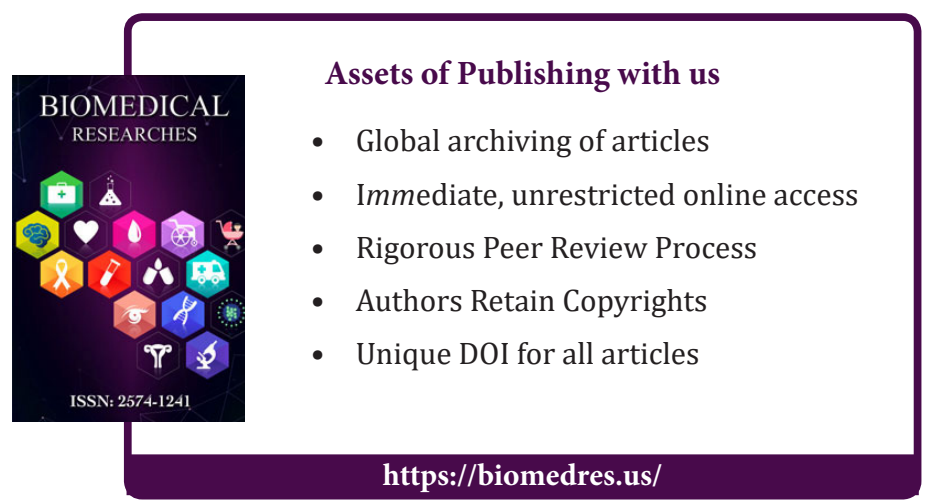

